Recibido:

junio 4 de 2021

Aceptado:

octubre 11 de 2021

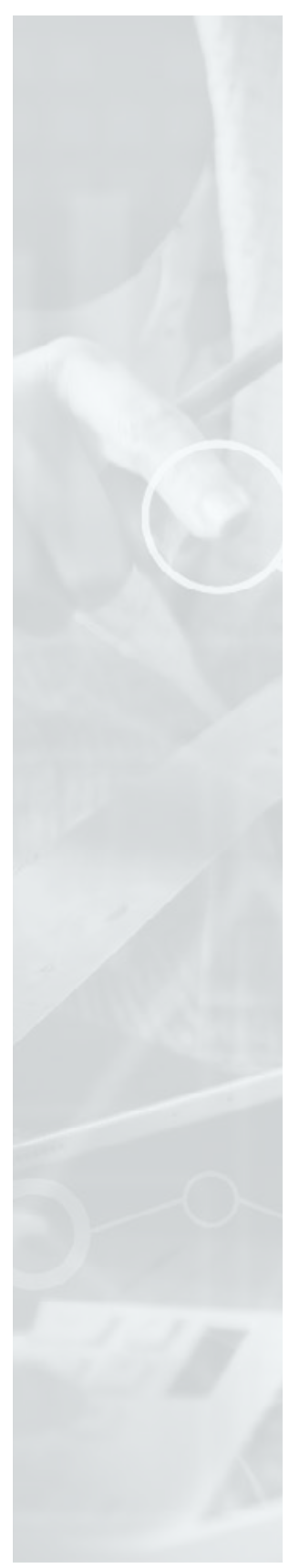

\section{La resiliencia como herramienta de superación en la gerencia de la empresa Multinacional de Seguros, C.A.}

\author{
Resilience as a tool for overcoming challenges \\ of the management of Multinacional de Seguros, C.A.

\begin{abstract}
Osmary Vanessa, Boadas Marquez1
osmaryboadas@gmail.com
\end{abstract} \\ https://orcid.org/0000-0003-1906-1797 \\ María Eugenia, Marín Mora2 \\ eugeniamarin.446@gmail \\ https://orcid.org/0000-0003-0697-2727 \\ https://doi.org/10.22209/amr.v1n1a01
}

\title{
Resumen
}

La investigación tuvo como objetivo analizar la aplicabilidad de la resiliencia por parte de la gerencia de la empresa Multinacional de Seguros, C.A, sucursal Ciudad Ojeda. El tipo de investigación fue analítica, con un diseño no experimental, transeccional y de campo. Como instrumento de recolección de datos se empleó la entrevista y como técnica, el guión de entrevista compuesto por 26 ítems, los cuales fueron validados bajo el juicio de expertos. El procesamiento de datos se realizó mediante el análisis cualitativo, el cual permitió concluir que en la organización objeto de estudio, la gerencia desarrolla satisfactoriamente la capacidad de resiliencia corporativa, resaltando que las acciones directivas han enfrentado con éxito algunos retos organizacionales tales como: generación de nuevo conocimiento en la ejecución de procesos, el enfoque estratégico, político e ideológico. Del mismo modo, se observó que, a través de la gestión ejecutiva, existe un

1 Facultad de Ciencias Administrativas, Universidad Alonso de Ojeda, Ciudad Ojeda, Edo Zulia Venezuela.

2 Facultad de Ciencias Administrativas, Universidad Alonso de Ojeda, Edo Zulia Venezuela. 
adecuado manejo de aspectos claves como: la mejora continua de procesos, adecuada estructura organizacional, idoneidad de sistemas administrativos y consolidación de la cultura organizacional; motivando ello a retomar los grupos de capacitación para la gerencia en tiempos de crisis, así como reanudar el desarrollo de actividades de motivación para impulsar en el personal el logro de sus objetivos.

Palabras clave: adaptabilidad; gerencia estratégica; multinacional de seguros; resiliencia.

\begin{abstract}
This research study aimed to analyze the application of resilience by the management of Multinacional de Seguros, C.A (Ciudad Ojeda site). The type of research was analytical with a non-experimental cross-sectional field design. The interview was used as the data collection instrument and a 26-item interview script was used as a technique, which was validated through expert judgment. Data processing was carried out through qualitative analysis, which led to conclude that in the organization under study the management has satisfactorily developed the capacity for corporate resilience. Managerial actions have successfully dealt with organizational challenges including generation of new knowledge in process execution, strategic approach, political approach and ideological approach. Likewise, it was observed that, through executive management, proper management of key aspects was implemented including: ongoing improvement of processes, adequate organizational structure, suitable administrative systems as well as consolidation of the organizational culture. This motivated to resume both the management training groups in times of crisis and the development of motivational activities to promote the achievement of goals among the staff.
\end{abstract}

Keywords: adaptability; strategic management; insurance multinational; resilience.

\title{
Introducción
}

Los seres humanos experimentan a diario circunstancias que les permiten demostrar sus capacidades de reaccionar, bien sea de forma positiva o negativa ante situaciones de riesgo, adversidad o dificultad. Esas capacidades impulsan al aprendizaje continuo, y es allí donde surge la resiliencia, cuyo significado está orientado a desarrollar la habilidad de seguir adelante a pesar de los problemas. Con la resiliencia se estudia cómo un individuo u organización están en capacidad de afrontar cambios constantes que se van presentando a lo largo del tiempo. 
La resiliencia hace énfasis entre adaptarse a un entorno turbulento o desaparecer; tener éxito o fracasar; se va cultivando a un ritmo ascendente para cuando sea necesario emplearla como una alternativa favorable. La resiliencia está vinculada a factores como la confianza, la seguridad de ajustarse y recuperarse frente a las adversidades, las cuales deben ser reconocidas como parte natural de la vida; siendo necesario encontrar las formas de manejar esas situaciones, afrontando los cambios que se dan en el entorno, y, sobre todo, saber cómo cultivar el aprendizaje e innovación para emerger entre las dificultades.

En ese sentido, las empresas desde su filosofía de gestión, tienen como propósito no sólo desarrollar su objeto social, sino, mantener un crecimiento sostenido que le lleve consigo a lograr una adecuada posición en el mercado, requiriendo para ello, contar con ventajas competitivas haciendo uso de un modelo de gestión basado en calidad del servicio. Es por ello, que las organizaciones resilientes se ven obligadas a reflexionar sobre lo que es necesario o no en términos de estrategias, estructura, sistemas internos y tendencias del mercado, permitiendo ello evaluar su propia capacidad instalada en criterios de eficiencia y efectividad, para así garantizar que el proceso de adaptabilidad se encamine correctamente.

En el orden de las ideas planteadas, actualmente en Venezuela, coexisten diversas empresas que, debido a la situación política, económica y social que se vive, su gestión operativa y financiera se ha visto afectada, sin embargo, éstas han tenido la necesidad de comenzar a desarrollar la resiliencia como la capacidad necesaria para sortear los obstáculos, alcanzar el éxito y consecuentemente, garantizar la durabilidad de sus negocios. Este tipo de empresas deben ser objeto de estudio para expandir y poner a prueba los conocimientos teóricos que existen en temas como la resiliencia; de ahí que la presente investigación, tuvo como propósito analizar la aplicabilidad de la resiliencia por parte de la gerencia de la empresa Multinacional de Seguros, C.A, valorando los retos organizacionales, aspectos claves y las etapas necesarias para el desarrollo de la misma.

\section{Fundamentación teórica}

El mundo actual está colmado de cambios inesperados y ambientes hostiles, lo cual ha generado como consecuencia que las empresas contemporáneas orienten su mirada hacia a atrás y evalúen con cuidado su entorno, asegurándose en reconocer las oportunidades y accionar para contrarrestar las amenazas, todo ello, haciendo buen uso de las fortalezas como factor interno de la misma unidad de negocio. Es así entonces, que surge como necesidad una estrategia gerencial que permita conducir la durabilidad y el éxito en el ciclo de vida de las organizaciones, que esté enfocada en la resistencia y una correcta adaptación al cambio. 
Los primeros estudios realizados sobre resiliencia, de los cuales se tiene evidencia, provienen de niños sobrevivientes a la Segunda Guerra Mundial, como es el caso de Boris Cyrulnik, considerado como el padre de la resiliencia acorde a los estudios de (Florentino, 2008 como se citó en León, 2013). Cyrulnik quedó huérfano a la edad de seis años, y a esa misma edad logró escapar de un campo de concentración nazi, para después reconstruir su vida, realizando estudios en las áreas de medicina y psicoanálisis. Desde entonces, el concepto de resiliencia se ha adoptado en diferentes disciplinas como la física, sociología, psicología y, más recientemente, en las ciencias sociales, específicamente en temas relacionados a la dirección de empresas.

Ahora bien, con relación a la presencia de la resiliencia en la dirección de empresas, Sutcliffe y Vogus (2003) señalan que la misma es la continuidad organizativa a partir de ajustes positivos que derivan de condiciones desafiantes, las cuales permiten que una organización emerja frente a un evento negativo, se fortalezca y optimice sus recursos. La resiliencia es una estrategia que brinda una ventaja competitiva sobre aquellas empresas que no lo son, sin embargo, resulta importante aclarar, que no es una capacidad fija; por el contrario, debe ser trabajada constantemente poniendo a prueba a los miembros de una organización y a la empresa como un todo.

Las empresas que cuentan con una estructura, estrategias y personal colaborador dispuesto a abordar los riesgos, son las que tienen la alta posibilidad de sobrevivir al final. De allí que grandes empresas hayan adoptado la resiliencia como estrategia para los desafíos organizacionales, demostrando que durante períodos de crisis existe una manera de seguir adelante mediante un plan estratégico apegado a su realidad y ajustado a sus recursos. En Latinoamérica también se han suscitado escenarios desfavorables debido a situaciones de índole política y social, tal es el caso de Venezuela, que actualmente, está atravesando por una coyuntura política, social, cultural y económica nunca antes vista en el país, por ello, los empresarios venezolanos se han visto en la necesidad de adaptarse e innovar, resistiendo en la adversidad sin dejar de cumplir con su misión.

En el Municipio Lagunillas del Estado Zulia, se encuentra ubicada la empresa Multinacional de Seguros, C.A., con más de veinticuatro (24) años en el mercado asegurador citojense, regional y nacional, que ha sido distinguida entre las más sólidas del sector asegurador, siendo también reconocida como una compañía que apoya la excelencia operacional, mediante la capacitación adecuada de sus miembros, fidelización de clientes, y planes estratégicos definidos.

Desde hace aproximadamente cinco años, la empresa se ha visto afectada por tres problemáticas principales, como lo son la migración elevada del talento 
humano nacional, el cierre de talleres asociados y migración de productores a otros campos del país o del exterior. Para poder contrarrestar el efecto negativo que la situación descrita ha generado, según conversaciones sostenida con la gerente de sucursal, la empresa en estudio se ha visto en la necesidad de crear condiciones propias de la resiliencia organizacional, a través del análisis de los retos a los que se enfrenta.

Como efecto del éxodo del recurso humano a nivel nacional, el volumen de trabajo ha incrementado para los que permanecen aún en la empresa, sin embargo, se ha creado un espacio que permite la retroalimentación de las diferentes áreas que la empresa maneja, lo cual ha impulsado el compromiso de los trabajadores fieles y consecuentes, que siguen brindando su apoyo, a la espera de un futuro más satisfactorio, promoviendo al mismo tiempo una cultura organizacional basada en apoyo, confianza y respeto. A pesar de la situación indeseable que atraviesa el país, la empresa ha sabido adaptarse a la coyuntura y se mantiene operativa con bajas ganancias, en una especie de punto de equilibrio, que le ha llevado también a mantenerse funcionando, en un nivel mínimo de operatividad, ventas e ingresos.

\section{Materiales y Métodos}

\section{Tipo y diseño de Investigación}

La presente investigación se consideró analítica puesto que tuvo como objetivo estudiar la resiliencia en la gerencia de la empresa Multinacional de Seguros, C.A., sucursal Ciudad Ojeda. Al respecto, Hernández et al. (2014) definen este tipo de investigación como aquel método de estudio que pone en práctica el pensamiento crítico y la evaluación de hecho y/o información pertinente a la investigación que se realiza, con el fin de encontrar los elementos esenciales que darán pie al análisis del tema seleccionado. Este tipo de trabajo le permitirá al investigador encontrar evidencia que apoye la investigación de manera que se pueda considerar fiable.

A su vez, respecto al diseño de una investigación, Atehortúa y Zwerg-Villegas (2012) señalan que se trata de una serie de métodos y técnicas elegidas y empleadas por el investigador al momento de realizar un proyecto de investigación, sirviendo como guía para ejecutarlo. El diseño de investigación consta de un conjunto de pasos o reglas concretas que permiten alcanzar el objetivo establecido para llevar a cabo el proyecto.

Dicho esto, la presente investigación se desarrolló mediante un diseño no experimental, es decir, sin modificar o manipular la variable de estudio; transeccional, 
por cuanto los datos fueron tomados en un único momento y finalmente, de campo debido a la información sujeta a análisis, fue recopilada en las instalaciones de la empresa objeto de estudio. Al respecto, Corbetta (2007) expone que los estudios no experimentales se basan en categorías, sucesos, variables o contextos que surgen sin la intervención directa del investigador, observando los acontecimientos tal y como se presentan en su contexto natural para ser analizados posteriormente.

En relación a la población, Arias (2012) la define como aquel conjunto finito o infinito de individuos con características similares para los cuales serán extensivas las conclusiones de la investigación, en ese sentido, la población de la presente investigación quedó conformada por tres (3) personas que forman parte del personal administrativo de la empresa Multinacional de Seguros, sucursal Ciudad Ojeda:

Tabla 1. Distribución poblacional

\begin{tabular}{|l|c|}
\hline \multicolumn{1}{|c|}{ Cargo } & Total \\
\hline Gerente de Sucursal & 1 \\
\hline Analista & 1 \\
\hline Perito & 1 \\
\hline \multicolumn{2}{|c|}{ Total } \\
\hline
\end{tabular}

Fuente: Departamento de Recursos Humanos. Multinacional de Seguros, C.A. (2020).

\section{Técnicas e instrumentos de recolección de datos}

La veracidad y efectividad de toda investigación siempre dependerá de la pertinencia de las técnicas que el investigador seleccione para la obtención de los resultados. Kuhn (1971), expone que estas conducen a la verificación del problema planteado, cada técnica establece sus herramientas, instrumentos o medios que serán empleados. En este proyecto de investigación se utilizó como técnica de recolección de datos la entrevista estructurada, la cual según Palella y Martins (2012) es una herramienta destinada a obtener datos de varias personas cuyas opiniones interesan al investigador, conduciendo a una comunicación bidireccional. En este trabajo se utilizó como instrumento el guión de entrevista, contentivo de 26 reactivos, cuyos resultados obtenidos fueron procesados a través del análisis cualitativo. Del análisis realizado surgió una matriz de resultados cualitativos que permitió la generalización de los datos obtenidos a fin de contrastarlos con las bases teóricas. 


\section{Resultados}

A continuación, se expone el análisis y la discusión de los resultados derivados del instrumento de recolección de datos aplicado a los tres (03) trabajadores que constituyeron la muestra dentro de la empresa Multinacional de Seguros, C.A., sucursal Ciudad Ojeda; los mismos son expuestos en una matriz de resultados cualitativos siguiendo el orden de la variable resiliencia, sus dimensiones e indicadores.

De acuerdo a la naturaleza del instrumento aplicado, se muestran tres (3) tablas con el resumen de los hallazgos obtenidos por dimensión, a fin de resaltar los datos importantes y relevantes para su mejor comprensión.

Tabla 2. Retos Organizacionales para la Resiliencia

\begin{tabular}{|c|c|c|}
\hline Dimensión & Indicadores & Hallazgos \\
\hline \multirow{14}{*}{$\begin{array}{l}\text { Retos } \\
\text { Organizacionales } \\
\text { para la resiliencia }\end{array}$} & \multirow{3}{*}{$\begin{array}{c}\text { Del } \\
\text { conocimiento }\end{array}$} & $\begin{array}{l}\text { El personal responsable de identificar los aspectos vitales corresponde a } \\
\text { cada trabajador en su área de trabajo. }\end{array}$ \\
\hline & & $\begin{array}{l}\text { La identificación de los aspectos vitales es un proceso sistemático de } \\
\text { comunicación donde el trabajador reporta al jefe de área y éste al } \\
\text { gerente de la sucursal. }\end{array}$ \\
\hline & & $\begin{array}{l}\text { Los parámetros operativos son la productividad de la sucursal (emisión y } \\
\text { renovación de pólizas), cartera de clientes y volumen de siniestros. }\end{array}$ \\
\hline & \multirow{4}{*}{ Estratégico } & $\begin{array}{l}\text { Quedó en desuso la vigencia anual en HCM, sustituyéndose por vigencias } \\
\text { bimensuales. }\end{array}$ \\
\hline & & $\begin{array}{l}\text { En automóvil, quedó en desuso la cobertura amplia, sustituyéndose por } \\
\text { pérdida total. }\end{array}$ \\
\hline & & En patrimoniales están en estudio las nuevas estrategias. \\
\hline & & $\begin{array}{l}\text { Los aspectos vulnerables para la empresa son: el no emitir pólizas en } \\
\text { dólares y la falta del recurso humano por el proceso de reducción de } \\
\text { costos en el que se encuentran. }\end{array}$ \\
\hline & \multirow{3}{*}{ Político } & La empresa no ha prescindido de personal, la fuerza laboral ha renunciado. \\
\hline & & No ofrecen mejoras en compensaciones económicas. \\
\hline & & $\begin{array}{l}\text { Las cualidades principales del personal son: abiertos al cambio, rápida } \\
\text { adaptación y constancia. }\end{array}$ \\
\hline & \multirow{4}{*}{ Ideológico } & La filosofía organizacional se mantiene intacta. \\
\hline & & $\begin{array}{l}\text { Las nuevas estrategias y el nuevo modo de operación se han alineado a la } \\
\text { filosofía establecida. }\end{array}$ \\
\hline & & $\begin{array}{l}\text { La empresa está dispuesta a asumir los cambios que sean necesarios en la } \\
\text { filosofía de gestión. }\end{array}$ \\
\hline & & La realidad del país está motivando a la empresa a redefinir su filosofía. \\
\hline
\end{tabular}

Fuente: elaboración propia, 2020. 
En la tabla 2 puede observarse que, en Multinacional de Seguros, C.A., está presente el reto del conocimiento, puesto que cada empleado es responsable de determinar cuáles son los factores importantes a encarar, sin esperar que alguien se los señale y cuando eso ocurre, debido al excelente proceso de comunicación que se acostumbra, se le informa al jefe inmediato.

En relación al reto estratégico, se evidencia que los empleados han sabido dejar atrás las prácticas que fueron efectivas, como la vigencia anual de las pólizas que han sido sustituidas por otras de duración bimensual y las pólizas de cobertura amplia para automóviles ahora son de pérdida total. Sin embargo, los aspectos en los que aún la empresa es vulnerable de acuerdo a este reto, es la negativa a emitir pólizas en dólares y la escasez de talento humano capacitado que se ha unido al éxodo generalizado en todo el país por la imposibilidad de la empresa de ofrecer sueldos competitivos.

En cuanto al reto político, se evidencia que la empresa no ha despedido a ninguno de los empleados a pesar de la situación indeseable que atraviesa, pero tampoco ofrecen mejores compensaciones económicas a los colaboradores que aún quedan. Otro aspecto relacionado con este reto es que, los empleados que activos poseen como cualidad, la capacidad de estar dispuestos a los cambios y a la adaptación. Finalmente, con respecto al reto ideológico, es importante señalar que Multinacional de Seguros. C.A., mantiene su filosofía inamovible, adaptando sus nuevas estrategias a la misma, aun con la disposición de afrontar los cambios que se le presenten.

Tabla 3. Aspectos claves de la resiliencia

\begin{tabular}{|c|c|c|}
\hline Dimensión & Indicadores & Hallazgos \\
\hline \multirow{6}{*}{$\begin{array}{l}\text { Aspectos claves } \\
\text { de la resiliencia }\end{array}$} & \multirow{3}{*}{ Estrategia } & Cambio de vigencias en pólizas de HCM y Automóvil. \\
\hline & & $\begin{array}{l}\text { Se han creado usuarios y contraseñas en el portal web a los } \\
\text { productores, para que estén atentos a información referente a estado } \\
\text { de solicitudes y pólizas. }\end{array}$ \\
\hline & & $\begin{array}{l}\text { Facilidad de pago directo a los clientes en un periodo de tiempo de tres } \\
\text { días. }\end{array}$ \\
\hline & \multirow{3}{*}{$\begin{array}{c}\text { Estructura } \\
\text { organizacional }\end{array}$} & Los departamentos y cargos se mantienen intactos. \\
\hline & & $\begin{array}{l}\text { Los trabajadores activos han asumido funciones de los cargos con } \\
\text { vacantes. }\end{array}$ \\
\hline & & $\begin{array}{l}\text { De } 40 \text { corredores, actualmente quedan sólo 15; de sociedad de } \\
\text { corretaje quedan } 2 \text { y de } 8 \text { de productores exclusivos, no queda } \\
\text { ninguno. }\end{array}$ \\
\hline
\end{tabular}




\begin{tabular}{|c|c|c|}
\hline Dimensión & Indicadores & Hallazgos \\
\hline \multirow{4}{*}{$\begin{array}{l}\text { Aspectos claves } \\
\text { de la resiliencia }\end{array}$} & \multirow{2}{*}{$\begin{array}{l}\text { Sistemas } \\
\text { administrativos }\end{array}$} & Los sistemas administrativos no se han cambiado. \\
\hline & & $\begin{array}{l}\text { Los trabajadores acuden a estos sistemas para conocer los lineamientos } \\
\text { de los cargos disponibles. }\end{array}$ \\
\hline & $\begin{array}{l}\text { Destreza } \\
\text { de cultura } \\
\text { organizacional }\end{array}$ & $\begin{array}{l}\text { Se contaba con capacitación para los líderes. Actualmente, se lleva a } \\
\text { cabo un seguimiento a través de reportes trimestrales para recursos } \\
\text { humanos. }\end{array}$ \\
\hline & & $\begin{array}{l}\text { Algunas de las habilidades desarrolladas por los líderes son: mentalidad } \\
\text { positiva, confianza en sí mismo, desarrollo de autoconsciencia al } \\
\text { estudiarse a sí mismo, y el mantenimiento de la higiene emocional. }\end{array}$ \\
\hline
\end{tabular}

Fuente: elaboración propia, 2020.

De acuerdo a lo señalado en la tabla anterior sobre la dimensión aspectos claves de la resiliencia, es menester señalar que, en cuanto a las estrategias, la empresa ha cambiado la vigencia de las pólizas para adecuarlas al poder adquisitivo de los posibles clientes. También se les ha dado una participación más directa a los productores, generándoles una contraseña en el portal web de la empresa, de modo que ellos mismos estén monitoreando las solicitudes de los clientes; estableciendo de igual manera mecanismos para otorgar facilidades de pago directo de siniestro en un lapso de hasta tres (3) días, dependiendo de las características del mismo.

Otro de los aspectos clave lo constituye la estructura organizacional, que durante los últimos años no ha sufrido cambios significativos, sin embargo, las funciones de los cargos que han quedado vacantes, son realizadas por el personal activo, generando ello un agotamiento físico, mental y emocional para los empleados que aún permanecen en sus funciones.

En relación al sistema administrativo, este tampoco ha sido modificado, lo que permite que los empleados desarrollen habilidades de autodidactas; permitiendo a su vez, que los colaboradores puedan encontrar en el sistema, los lineamientos de los cargos que les ha tocado ejercer simultáneamente, y como aspecto clave final, se puede mencionar lo relacionado a la cultura organizacional, enfocada a la capacitación y el entrenamiento sistemático, el cual se ha dejado de ofrecer a los empleados y ha sido erróneamente sustituido por actividades de seguimiento al desempeño mediante reportes trimestrales entregados al departamento de recursos humanos, así como por una solicitud implícita para que el personal se auto evalúe y auto motive para mantener el ánimo y la actitud positiva. 
Tabla 4. Etapas de la resiliencia

\begin{tabular}{|c|c|c|}
\hline Dimensión & Indicadores & Hallazgos \\
\hline \multirow{11}{*}{$\begin{array}{l}\text { Etapas de la } \\
\text { resiliencia }\end{array}$} & \multirow{6}{*}{ Detección } & $\begin{array}{l}\text { La realidad política, económica, social y cultural del país como } \\
\text { factor impulsor de la resiliencia. }\end{array}$ \\
\hline & & Montos elevados de siniestros en HCM y Automóvil. \\
\hline & & Imposibilidad de mantener la vigencia anual. \\
\hline & & Montos elevados y cambiantes en baremos de clínicas. \\
\hline & & Presupuestos susceptibles al cambio continuo. \\
\hline & & $\begin{array}{l}\text { Los riesgos encarados fueron desacuerdos entre clientes y } \\
\text { productores, bajo poder adquisitivo, pérdida de clientes. }\end{array}$ \\
\hline & \multirow[t]{2}{*}{ Activación } & $\begin{array}{l}\text { Necesidad de afianzar la relación entre proveedores, corredores y } \\
\text { sociedades de corretaje mediante visitas directas. }\end{array}$ \\
\hline & & Necesidad de conversaciones con los clientes directos en oficinas. \\
\hline & \multirow[b]{2}{*}{ De respuesta } & Baja tasa en pérdida de clientes. \\
\hline & & $\begin{array}{l}\text { Se ha mantenido un volumen en la cartera de clientes que permite } \\
\text { su funcionamiento y cumplimiento de objetivos. }\end{array}$ \\
\hline & $\begin{array}{c}\text { Aprendizaje } \\
\text { organizacional }\end{array}$ & $\begin{array}{l}\text { Importancia del trabajo en equipo, nivel de adaptación al cambio, } \\
\text { búsqueda de soluciones rápidas y efectivas, alta relevancia al } \\
\text { servicio directo y fidelización de clientes y proveedores. }\end{array}$ \\
\hline
\end{tabular}

Fuente: elaboración propia, 2020.

Ahora bien, respecto a la tercera y última dimensión relacionada a la etapa de la resiliencia, de acuerdo a lo señalado por el personal entrevistado, se pudo verificar que, entre los cambios del entorno se encuentra el incremento del costo de los siniestros de las pólizas Hospitalización Cirugía y Maternidad (HCM) y de automóviles, llevando a la gerencia a no poder mantener vigentes los precios durante el tiempo normalmente establecido en los contratos de servicios según las políticas organizacionales. En relación a la segunda fase de la resiliencia, la activación, la gerencia mediante visitas y reuniones constantes para poder establecer las estrategias de subsistencia, tuvo la oportunidad de consolidar la relación entre proveedores, corredores, sociedades de corretaje y con los propios clientes. La tercera etapa se corresponde a la de respuesta, sobre la cual se evidenció la disminución acelerada de clientes que llegó al mínimo requerido para sufragar los costos operativos en punto de equilibrio; finalmente, como última etapa, el aprendizaje organizacional, puso en relieve la importancia del trabajo en equipo, frente a la necesidad imperiosa de adaptación a la nueva realidad nacional y sobre todo, a la búsqueda de soluciones rápidas pero efectivas para fidelizar tanto a clientes como a proveedores. 


\section{Discusión}

Una vez analizados los resultados obtenidos de la entrevista estructurada, se procede a la discusión de los resultados y a su contrastación con la teoría que sustenta este trabajo de investigación. En relación a la primera dimensión relacionada a la identificación de los retos organizacionales: generación de conocimiento, enfoque estratégico, político e ideológico, los resultados demuestran la capacidad gerencial que le ha permitido a la empresa mayor longevidad en el mercado, determinando consigo la posibilidad de reacción y respuesta tanto a la gerencia como de la fuerza laboral en el contexto actual.

En ese sentido, frente a los retos que exige el proceso resiliente dentro de la organización, se puede referir que los resultados obtenidos señalan que los mismos están alineados con lo que plantea Schneider (2007), "la excitación por el cambio debe ser permanente pero nunca traumática; debe haber un reacomodo continuo y no cambios traumáticos, debe tratarse más de una evolución que de una revolución" (p.112). Asimismo, se añade que cualquier organización que busque la resiliencia debe enfrentar con éxito los retos del conocimiento, estratégico, político e ideológico.

En lo que se refiere a la segunda dimensión, aspectos claves de la resiliencia que maneja la gerencia de la empresa Multinacional de Seguros, C.A., sucursal Ciudad Ojeda, se identificaron cuatro aspectos: la estrategia, estructura organizacional, sistemas administrativos y destreza de cultura organizacional, los cuales, bajo un manejo adecuado, le permiten a los trabajadores y a la empresa asumir cambios para adaptarse a las necesidades del mercado. En ese sentido, vale acotar que los resultados obtenidos coinciden totalmente con lo que nuevamente señala Schneider (2007), cuando refiere que para que una organización resista en tiempos de inestabilidad y responda con éxito a los retos, es necesario que maneje las áreas o aspectos claves de la resiliencia.

En el orden de las ideas planteadas en el párrafo anterior, Kotliarenco et al. (1997), refieren que la resiliencia abre un abanico de posibilidades, en tanto enfatiza las fortalezas y aspectos positivos presentes en los seres humanos; lo cual a su vez para los investigadores significa una oportunidad en el ámbito empresarial, por cuanto, en la medida a que los colaboradores sean capaces de desarrollar habilidades que le permitan sortear sus obstáculos, ello será de gran valor para la toma de decisiones gerenciales y el alcance de los resultados. Por su parte, para Bozz (2004), las organizaciones hoy día están llamadas a integrar la resiliencia en la cultura corporativa, debido a que ello le permitirá entrar en la búsqueda constante de ideas claves para prever y resolver los tiempos de crisis. 
Finalmente, en relación a la tercera dimensión, etapas de la resiliencia aplicadas en la gerencia de la empresa Multinacional de Seguros, C.A., sucursal Ciudad Ojeda, se evidenció que la misma aplica las siguientes etapas: detección, activación, de respuesta y aprendizaje organizacional, las cuales son cualidades inherentes de las unidades de negocios que se permiten responder con éxito en periodos críticos. Los resultados obtenidos en esta última dimensión, están respaldados por la opinión de Chirinos et al. (2018) quienes señalan que una organización resiliente tiene que tener una capacidad adaptativa, en donde la posibilidad de reestructurarse oportunamente, es la clave para responder a eventos emergentes. Por su parte, Medina (2012), plantea que en las organizaciones se requiere una voluntad corporativa que sea capaz de dinamizar el compromiso para gestionar todos los recursos que sean necesarios para poder reconocer y enfrentar los problemas que coexisten en el entorno cambiante.

También son coincidentes los resultados con la postura de Bjamra, Dani y Burnard (2011), quienes sostienen que las etapas de la resiliencia son las fases de respuesta que una organización desarrolla durante un momento o situación crítica, a las que consideran cualidades adaptativas de una organización para responder con éxito los períodos críticos.

\section{Conclusiones}

En virtud de los resultados obtenidos en la presente investigación, luego de la aplicación del instrumento de recolección de datos, se presentan las conclusiones del estudio en función a cada una de las dimensiones relacionadas al objeto de estudio. En primer lugar, se hará referencia a la dimensión retos organizacionales para la resiliencia por parte de la gerencia de la empresa Multinacional de Seguros, C.A., sucursal Ciudad Ojeda.

Mediante el indicador de conocimiento, se evidenció que la empresa identifica los aspectos vitales que le servirán para hacer frente a situaciones turbulentas a través de un sistema en el cual cada trabajador es el encargado de identificar las necesidades en su área y comunicarlas al jefe directo; lo antes señalado bajo parámetros de producción previamente definidos como: la cartera de clientes, el volumen de siniestros y el nivel de producción de emisión y renovación de pólizas, los cuales son elementos que ofrecen una idea de la posición futura de la unidad de negocios.

Asimismo, la información obtenida en el indicador estratégico, demuestra que el reemplazo de las estrategias antiguas por nuevas acciones directivas en cuanto a vigencias y coberturas de pólizas, evidencia una reinvención de las mismas como 
respuesta a las nuevas realidades del entorno. De igual manera, se muestra que la empresa no es ajena a tener aspectos vulnerables en el proceso de resiliencia, siendo éstos la incapacidad de emitir pólizas en divisas y la falta de recursos humanos por reducción de costos.

Por otra parte, el indicador político ha puesto en evidencia que, como resultado de la crisis, gran parte del personal trabajador se ha retirado voluntariamente, lo que en consecuencia ha generado que la fuerza laboral que permanece tenga capacidad adaptativa; desarrollen actitudes positivas frente a los cambios; sepan adaptarse a ellos y practiquen la cultura de trabajo como cualidades durante el proceso de regeneración operativa.

A su vez, el indicador ideológico refleja que la filosofía organizacional no ha sido cambiada durante el proceso de resiliencia por cuanto es considerada como sólida, de modo que tampoco la empresa se ha visto en la necesidad de cambiarla por la situación actual, sin embargo, se muestran dispuestos a realizar cambios si, en algún momento en el futuro, las circunstancias del país les conllevan a ello.

Con respecto a la segunda dimensión, áreas claves de la resiliencia que maneja la gerencia de la empresa Multinacional de Seguros, C.A, sucursal Ciudad Ojeda, se identificó la estrategia, estructura organizacional, sistemas administrativos y destreza de cultura organizacional como áreas claves. Mediante el indicador estrategia se demostró que las acciones aplicadas por la empresa relativas a cambio de vigencia de pólizas, facilidad de pago y uso del portal web, han sido realizadas en orden de asegurar el éxito del proceso de resiliencia.

En lo relativo a la estructura organizacional, esta no se ha visto afectada, por lo que los departamentos y cargos se mantienen intactos, trayendo consigo que los trabajadores activos asuman las funciones de los cargos vacantes para asegurar el funcionamiento de la empresa. También se evidencia que los sistemas administrativos no han sido cambiados ni modificados, pese a que el modo de operación no es el mismo; estos han servido como una guía para que los colaboradores puedan reconocer el funcionamiento de la empresa en los lineamientos que requieran, coadyuvando así al proceso de resiliencia.

Con relación, a la destreza de cultura organizacional, se muestra que fue necesario establecer capacitación a los líderes para afinar su estilo gerencial con la fuerza laboral, lo que, a su vez, permitió desarrollar habilidades en los líderes como una mentalidad positiva, para poder conectar con confianza, el desarrollo de autoconsciencia y el mantenimiento de la higiene emocional que brinda la energía requerida para estar en un proceso de reestructuración. 
En el mismo orden de ideas, en cuanto a la dimensión etapas de la resiliencia aplicadas en gerencia de la empresa Multinacional de Seguros, C.A., se determinó la detección, activación, respuesta y aprendizaje organizacional, como etapas de la variable de estudio. Se logró evidenciar que la detección es una etapa aplicada en la empresa que contribuye a la preparación de estrategias mediante la identificación de amenazas reales en el entorno, como lo son los montos elevados de siniestros, los montos elevados y cambiantes de baremos de clínicas, y presupuestos de talleres, entre otros. De igual modo, en la activación, la empresa realizó un proceso para fidelizar a los clientes y proveedores, a fin de tomar acciones para no suspender las actividades en el momento en que las estrategias para combatir las amenazas detectadas fueran aplicadas.

Finalmente, frente al indicador de respuesta se demostró que, pese a que en una primera instancia se obtuvo pérdida de clientes; la tasa de pérdida no fue lo suficientemente alta para suponer un riesgo o amenaza potencial, por cuanto las estrategias de la empresa le han permitido seguir con su funcionamiento manteniendo un nivel de producción estable. En cuanto al aprendizaje organizacional, la empresa ha adquirido conocimientos que ha puesto en práctica a raíz de la relevancia de ellos durante el proceso de resiliencia, la importancia del trabajo en equipo, la búsqueda de soluciones que sean efectivas y la vitalidad de mantener un servicio directo con los clientes - proveedores, a fin de fidelizar los mismos y fortalecer la relación comercial de la empresa.

\section{Referencias}

Arias, F (2012). El Proyecto de Investigación. Editorial Episteme.

Atehortúa, F. y Zwerg-Villegas, A. (2012). Metodología de la investigación: más que una receta. $A D$-minister, 20, 91-111.

Booz, A. (2004). Redefiniendo la agenda del gobierno corporativo de la administración del riesgo a la resiliencia empresarial. The Australian journal of emergency Management. www.boozallen.com/media/file/Redefining_Corp_Gov_Agen

Bhamra, R., Dani, S., \& Burnard, K. (2011). Resilience: the concept, a literature review and future directions. International journal of production research, 49(18), 5375-5393.

Chirinos, J. y Arias, A. (2018). Resiliencia Organizacional y Gestión de Riesgos. Caracas, Venezuela. https://www.pwc.com/ve/es/publicaciones/assets/PublicacionesNew/ Estudios/1ra_Encuesta_Resiliencia_Riesgos_2018.pdf

Corbetta, P (2007). Metodología y Técnicas de Investigación Social. Editorial Mc Graw Hill, Madrid España.

Hernández, R., Fernández, C. y Baptista, P. (2014). Metodología de la investigación. Editorial Mc Graw-Hill. 
Kotliarenco, M. A., Cáceres, I., \& Fontecilla, M. (1997). Estado de arte en resiliencia. Organización Panamericana de la salud.

Kuhn, T (1971). La estructura de las revoluciones científicas. Fondo de Cultura Económica.

León, P. (2013). Resiliencia Organizacional: Una Aproximación. Universidad Del Rosario. Bogotá, Colombia.

Medina Salgado, C. (2012). La resiliencia y su empleo en las organizaciones.

Palella, S. y Martins F. (2012). Metodología de la Investigación Cuantitativa. Editorial FEDUPEL.

Schneider, B. (2007). Resiliencia. Editorial Norma.

Sutcliffe, K. \& Vogus, T. (2003). Organizing for Resilience In K. S. Cameron, J. E. Dutton\& R. E. Quinn (Eds.), Positive organizational scholarship: Foundations of a new discipline (pp. 94-110). San Francisco: Berrett-Koehler. 